\title{
miRNA-200c increases the sensitivity of breast cancer cells to doxorubicin through the suppression of E-cadherin-mediated PTEN/Akt signaling
}

\author{
YONG CHEN $^{1 *}$, YING SUN $^{2 *}$, LONGBANG CHEN $^{3}$, XINGXIANG XU $^{4}$, \\ XIZHI ZHANG ${ }^{1}$, BUHAI WANG ${ }^{1}$, LINGFENG MIN ${ }^{4}$ and WEI LIU ${ }^{5}$ \\ ${ }^{1}$ Department of Medical Oncology, Subei People's Hospital, Yangzhou, Jiangsu 225000; ${ }^{2}$ Department of Pathology,
Hebei United University of Basic Medical Sciences, Tangshan, Hebei 063009; ${ }^{3}$ Department of Medical Oncology,
Jinling Hospital, School of Medicine, Nanjing University, Nanjing, Jiangsu 210002;
${ }^{4}$ Department of Respiratory Medicine, Subei People's Hospital, Yangzhou, Jiangsu 225000;
Department of Medical Oncology, Hebei Medical University Fourth Hospital, Shijiazhuang, Hebei 050011, P.R. China
}

Received December 18, 2012; Accepted February 20, 2013

DOI: $10.3892 / \mathrm{mmr} .2013 .1403$

\begin{abstract}
Doxorubicin (ADR) is successfully used to treat breast cancer, however, it is often associated with the acquired resistance of breast cancer cells which eliminates the therapeutic efficiency of ADR, leading to relapse and a poorer prognosis. It has been reported that microRNA-200c (miRNA-200c), a non-coding RNA, is important in the epithelial to mesenchymal transition (EMT) and metastasis in breast cancer cells. Recent evidence demonstrated that miRNA$200 \mathrm{c}$ is also regulated in chemotherapeutic drug resistance, however, the precise mechanism by which this occurs remains unclear. In this study, we demonstrated that the loss of miRNA-200c correlates with the acquired resistance of breast cancer cells to ADR. In addition, the loss of miRNA-200c correlated with decreased levels of E-cadherin and PTEN, and increased levels of ZEB1 and phospho-Akt (p-Akt) in ADR-resistant breast cancer cells (MCF-7/ADR cells). More importantly, we demonstrated that the gain of miRNA-200c results in an increased sensitivity of cells to ADR, downregulation of ZEB1, upregulation of E-cadherin and PTEN, and inactivation of Akt signaling. Following the co-transfection of E-cadherin siRNA, the miRNA-200c-mediated regulation of Akt signaling and PTEN was inhibited. Results of the present study also demonstrated that Akt signaling is involved in the ADR resistance of breast cancer cells since LY294002, an
\end{abstract}

Correspondence to: Professor Wei Liu, Department of Medical Oncology, Hebei Medical University Fourth Hospital, No. 12 Jiankang Road, Shijiazhuang, Hebei 050011, P.R. China E-mail: hebeiliuwei@yahoo.com.cn

*Contributed equally

Key words: miRNA-200c, E-cadherin, PTEN, Akt, doxorubicin resistance, breast cancer inhibitor of Akt signaling, partially restored the sensitivity of MCF-7/ADR cells to ADR. In conclusion, miRNA-200c inhibited Akt signaling through its effects on E-cadherin and PTEN, resulting in the inhibition of ADR resistance in breast cancer cells.

\section{Introduction}

Breast cancer is the leading cause of cancer-related mortality in the female population and chemotherapy alone or combined with surgery currently forms the mainstay of clinical treatment. In anthracycline-containing regimens, doxorubicin (ADR) is considered as the main choice in chemotherapy for the treatment of breast cancer since it effectively reduces the annual probability of recurrence and mortality, particularly for high-risk breast cancer. However, acquired resistance to ADR currently forms a major obstacle to successful treatment.

The causes of cancer-specific drug resistance are currently believed to be associated with epigenetic gene changes, including DNA methylation (1-3). Extensive studies have indicated the existence and importance of another mechanism of non-mutational regulation of gene function, which is mediated by microRNAs (miRNAs). miRNAs are noncoding RNAs that mainly repress post-transcriptional gene expression, causing the inhibition or degradation of mRNA translation (4). miRNA-200c, a member of the miRNA-200 family, has been shown to be downregulated in a variety of human cancer types (5-7). A study by Cochrane et al reported that chemosensitivity to paclitaxel was significantly increased following the transfection of miRNA-200c in endometrial cancer cells (8). Similarly, Ceppi et al observed that the upregulation of miRNA-200c restored the sensitivity of non-small cell lung cancer (NSCLC) cells to cisplatin and cetuximab (9). These results indicate that miRNA-200c is important in inhibiting the development of drug resistance in cancer cells. miRNA-200c is also aberrantly expressed in breast cancer cells with an ADR-resistant phenotype (10), indicating that a decrease in miRNA-200c expression is 
linked to ADR resistance in breast cancer cells. However, the significance and precise molecular mechanism by which this occurs remains unclear.

Phosphatase and tensin homolog (PTEN) is a dualspecificity phosphatase, dephosphorylating lipid and protein substrates, and is important in the suppression of tumor cell proliferation and cell migration, signaling and apoptosis (11). Downregulation or mutation of the PTEN gene is frequently observed in numerous types of human cancer, including breast, prostate, brain, melanoma and glioma. Recently, in a study aimed to investigate the acquired resistance mechanisms to cetuximab in NSCLC, Kim et al demonstrated that PTEN protein levels were decreased in cetuximabresistant cell lines (HCC827-CR), leading to the activation of phosphoinositide 3-kinase (PI3K)/Akt signaling, while the upregulation of PTEN significantly reduced Akt activity and restored drug sensitivity, indicating that PTEN downregulation promoted the acquired resistance of NSCLC to cetuximab by activation of PI3K/Akt signaling (12). A previous study has shown that PTEN levels are regulated by E-cadherin, which is one of the most important cell adhesion molecules in epithelial cells and considered to function downstream of miRNA-200c. Restoration of miRNA-200c activity directly targets and downregulates zinc finger E-boxbinding homeobox 1 (ZEB1), thereby increasing E-cadherin expression and subsequently leading to EMT repression (13). In addition, the loss of E-cadherin in ovarian cancer cells downregulated PTEN expression via $\beta$-catenin-mediated Egrl regulation, contributing to the activation of PI3K/Akt signaling and cell proliferation (14). On the basis of these previous findings, we hypothesize that downregulation of miRNA-200c-dependent E-cadherin/PTEN signaling may lead to the activation of PI3K/Akt, which subsequently regulates the ADR resistance of breast cancer cells.

In this study, we aimed to demonstrate that miRNA-200c inhibits the acquired resistance of breast cancer cells against ADR via inactivation of the PI3K/Akt signaling pathway. Upregulation of miRNA-200c results in an increase in the expression of E-cadherin through inhibition of ZEB1 and a decrease in phosphorylated Akt levels by upregulation of PTEN. By contrast, miRNA-200c-upregulated PTEN levels are reduced when cells are co-transfected with E-cadherin siRNA, leading to re-activation of the PI3K/Akt signaling pathway. Furthermore, decreased Akt activity increases the sensitivity of breast cancer cells to ADR. Our results indicate that an interplay between the miRNA-200c assembly and Akt signaling exists, which may provide important insights into the role of miRNA-200c in ADR resistance.

\section{Materials and methods}

Cell culture. The human breast cancer MCF-7 and MCF-7/ ADR (resistant to ADR) cell lines were obtained from the Hebei Province Cancer Institute (Hebei, China) and cultured in RPMI-1640 medium supplemented with $10 \%$ fetal calf serum (Gibco BRL, Grand Island, NY, USA) in a humidified atmosphere containing $5 \% \mathrm{CO}_{2}$ at $37^{\circ} \mathrm{C}$. For MCF-7/ADR cells, ADR (Sigma, St. Louis, MO, USA) was additionally added at a final concentration of $1 \mathrm{mg} / 1$ to maintain the resistance phenotype until one week prior to the experiments.
Cell transfection. MCF-7/ADR cells were plated in 6-well plates $\left(4 \times 10^{5}\right.$ cells/well) and transfected with $20 \mathrm{nM}$ of either miRNA-200c precursor (Applied Biosystems, Carlsbad, CA, USA) or a mixture of miRNA-200c precursor and E-cadherin siRNA (20 $\mathrm{nM}$, reference sequence: 5'-CAGACAAAGACCAGGACUA-3', Genepharma, Shanghai, China) using Lipofectamin ${ }^{\mathrm{TM}} 2000$ transfection reagent and Opti-MEM I-reduced serum medium (Invitrogen, Carlsbad, CA, USA) according to the manufacturer's instructions. Cells transfected with scrambled RNA oligonucleotide served as a control. At $24 \mathrm{~h}$ post-transfection, cells transfected with miRNA-200c precursor were harvested for drug sensitivity assay and quantitative real-time polymerase chain reaction (qRT-PCR).

Measurement of cell sensitivity to ADR. The MTT [3-(4,5-dimethyl-2-thiazolyl)-2,5-diphenyl-2H-tetrazolium bromide] assay was used to determine drug sensitivity. MCF-7 and MCF-7/ADR cells with cell transfection or treatment with Akt inhibitor LY294002 were plated in 96-well plates at a concentration of $7 \times 10^{3}$ viable cells/well and cultured overnight. On the following day, medium was replaced with fresh medium containing different concentrations of ADR (final concentration: $0.1,1,10$ and $100 \mu \mathrm{g} / \mathrm{ml}$ ) and cells were incubated for $48 \mathrm{~h}$. Cells were then cultured for $4 \mathrm{~h}$ with $20 \mu \mathrm{l}$ of MTT (5 mg/l, Sigma) and $150 \mu \mathrm{l}$ of DMSO. Absorbance was read at $490 \mathrm{~nm}$ using a microplate spectrophotometer (Bio-Rad Laboratories, Hercules, CA, USA). The IC50 value, defined as the drug concentration at which cell survival was reduced to $50 \%$, was assessed by the relative viability curve according to the absorbance of MTT.

qRT-PCR analysis for miRNA-200c expression. Total RNA was extracted from cells using TRIzol (Invitrogen) according to the manufacturer's instructions. After $1 \mu \mathrm{g}$ of total RNA was reverse transcribed into cDNA, qRT-PCR was performed using SYBR-Green PCR mastermix (Invitrogen). The primers were synthesized by Shenggong Biotech Company Ltd. (Shanghai, China) and the sequences of the miRNA-200c primers were: forward, 5'-GGTAATACTGCCGGGTAAT-3' and reverse, 5'-CAGTGCTGTCGTGAGT-3'. Thesequences of theU6primers were: forward, 5'-GCTTCGGCAGCACATATACTAAAAT-3' and reverse, 5'-CGCTTCACGAATTTGCGTGTCAT-3'. PCR was performed using a thermal cycler (Rotor-Gene 3000, Corbett Research, Australia) and the conditions were as follows: denaturation at $95^{\circ} \mathrm{C}$ for $5 \mathrm{~min}$, followed by 40 cycles for $10 \mathrm{sec}$ at $95^{\circ} \mathrm{C}, 20 \mathrm{sec}$ at $60^{\circ} \mathrm{C}$ and $20 \mathrm{sec}$ at $78^{\circ} \mathrm{C}$. The level of miRNA-200c was calculated using the $2^{-\Delta \mathrm{Ct}}$ method, where $\Delta \mathrm{Ct}$ was the difference in threshold cycles for target and reference $=\mathrm{Ct}_{\text {miRNA-200c }}-\mathrm{Ct}_{\mathrm{U} 6}$ and expressed by the fold changes relative to $\mathrm{MCF}-7 / \mathrm{ADR}$.

Western blot analysis. MCF-7 and MCF-7/ADR cells with cell transfection or treatment with Akt inhibitor LY294002 were collected and homogenized in lysis buffer $(50 \mathrm{mM}$ Tris- $\mathrm{HCl}$, $\mathrm{pH} 8.0,150 \mathrm{mM} \mathrm{NaCl}, 0.1 \%$ SDS, $1 \%$ Nonidet P-40, $0.5 \%$ sodium deoxycholate, $0.02 \%$ sodium azide, $100 \mathrm{mg} / \mathrm{l} \mathrm{PMSF}$, $1 \mathrm{mg} / \mathrm{l}$ aprotinin) and centrifuged at $14,000 \mathrm{x} \mathrm{g}$ for $10 \mathrm{~min}$. The supernatant was collected and the total protein content was determined using the Bradford assay. The proteins (50 $\mu \mathrm{g} / \mathrm{lane})$ 


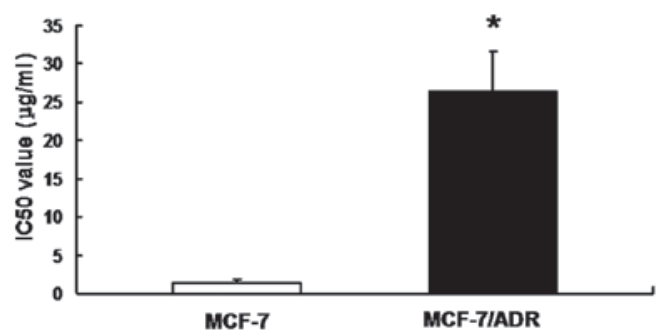

Figure 1. ADR sensitivity in MCF-7 and MCF-7/ADR cells as indicated by IC50 values (ADR concentration that induces 50\% cell death). The IC50 value is determined using the MTT assay. ${ }^{*} \mathrm{P}<0.01$ compared with MCF-7 cells. MTT, 3-(4,5-dimethyl-2-thiazolyl)-2,5-diphenyl-2H-tetrazolium bromide; ADR, doxorubicin.

were separated on $10 \%$ SDS-PAGE gels and transferred to PVDF membranes (Bio-Rad). Membranes were blocked with $5 \%$ non-fat milk and incubated overnight at $4{ }^{\circ} \mathrm{C}$ with primary antibodies against ZEB1, E-cadherin, PTEN, phosphoAkt (p-Akt, Ser473), total Akt and $\beta$-actin (Santa Cruz Biotechnology, Inc., Santa Cruz, CA, USA). The concentration of antibodies was 1:200 for ZEB1, E-cadherin, PTEN, total Akt and p-Akt, and 1:1000 for $\beta$-actin. Following washing, the membranes were incubated with infrared dye-labeled secondary antibodies, IRDyeTM800DX-conjugated affinitypurified anti-mouse IgG or IRDyeTM700DX-conjugated affinity-purified anti-rabbit IgG (1:10000 dilution, LI-COR Biosciences, Lincoln, NE, USA). Target bands were visualized using the Odyssey ${ }^{\circledR}$ Infrared imaging system (version 3.0, LI-COR Biosciences). The results of ZEB1, E-cadherin and PTEN were expressed as the ratio of the density of specific bands compared with $\beta$-actin, while p-Akt was normalized by total Akt.

Statistical analysis. Experiments were performed in triplicate. Data were presented as the mean \pm standard deviation (SD) and were analyzed with the Student-Newman-Keuls t-test using SPSS 13.0 software (Chicago, IL, USA). $\mathrm{P}<0.05$ was considered to indicate a statistically significant result.

\section{Results}

MCF-7/ADR cells demonstrated resistance to ADR. To detect the drug resistance of MCF-7/ADR cells, the in vitro sensitivity of MCF-7/ADR cells to ADR was evaluated by MTT assay. Our results demonstrated that the IC50 value of MCF-7/ ADR was $26.548 \pm 5.078 \mu \mathrm{g} / \mathrm{ml}$, while the value for MCF-7 alone was $1.364 \pm 0.494 \mu \mathrm{g} / \mathrm{ml}$, suggesting that MCF-7/ADR cell lines possessed a strong resistance to ADR (Fig. 1).

Restoration of miRNA-200c increased the drug sensitivity of MCF-7/ADR cells to ADR. To determine the underlying mechanism for the development of acquired ADR resistance in breast cancer cells, the expression of miRNA-200c was initially detected using qRT-PCR. As shown in Fig. 2A, MCF-7/ADR cells exhibited decreased levels of miRNA-200c expression compared with its parental MCF-7 cells. miRNA200c precursor was transfected into MCF-7/ADR cells and miRNA-200c expression was detected as well as sensitivity to ADR. The results showed that transfection signifcantly
A

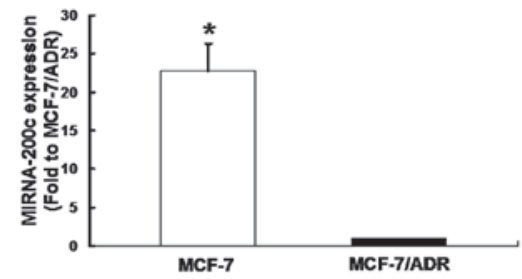

B

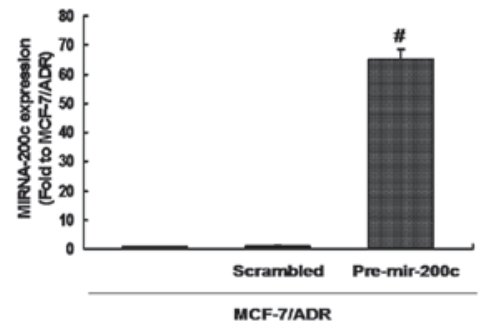

C

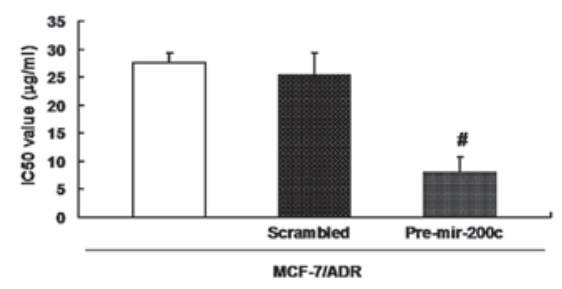

Figure 2. Effect of miRNA-200c levels on ADR sensitivity in MCF-7/ADR cells. (A) miRNA-200c expression levels are decreased in MCF-7/ADR cells. miRNA-200c is measured by qRT-PCR analysis and normalized by U6 snRNA. The results are presented as the fold change of miRNA-200c of MCF-7 compared with MCF-7/ADR cells. (B) Transfection with miRNA-200c precursor (pre-mir-200c) leads to an increase in the levels of miRNA-200c in MCF-7/ADR cells. (C) Transfection with pre-mir-200c restores the ADR sensitivity in MCF-7/ADR cells as the IC50 value decreases. " $\mathrm{P}<0.01$ compared with MCF-7/ADR cells, ${ }^{\text {P }}<<0.01$ compared with scrambled control. ADR, doxorubicin; qRT-PCR, quantitative real-time polymerase chain reaction; miRNA-200c, microRNA-200c.

upregulated miRNA-200c expression in MCF-7/ADR cells (Fig. 2B). Notably, the drug sensitivity assay revealed that the IC50 value following transfection of miRNA-200c precursor was markedly lower compared with transfection with scrambled RNA oligonucleotide, indicating that the sensitivity of MCF-7/ADR to ADR was restored (Fig. 2C). These data show that the drug resistance of MCF-7/ADR cells was partially due to the decreased expression of miRNA-200c.

Low miRNA-200c expression correlated with low levels of E-cadherin protein and upregulation of ZEBI expression. It is well established that cellular resistance to chemotherapeutic drugs correlates with the loss of E-cadherin expression (15) and recent studies have demonstrated that miRNA-200c may be capable of upregulating E-cadherin expression through direct targeting of its ZEB1 transcriptional repressors, as a result of the miRNA-200c-binding site being located within the ZEB1 $3^{\prime}$ untranslated region (UTR) in breast cancer cells $(13,16,17)$. In the present study, we determined whether a negative correlation between miRNA-200c and ZEB1 in MCF-7/ADR cells exists. Consistent with previous studies (13), our results demonstrated high levels of ZEB1 expression in MCF-7/ADR cells with low levels of miRNA-200c expression. Since ZEB1 is a potent repressor of E-cadherin, our results also showed 

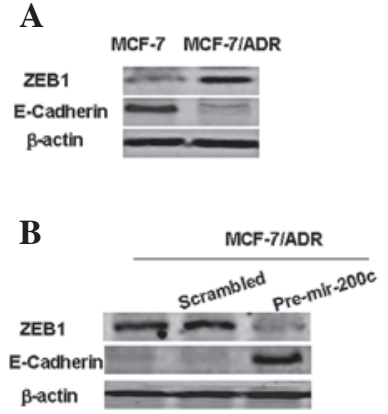
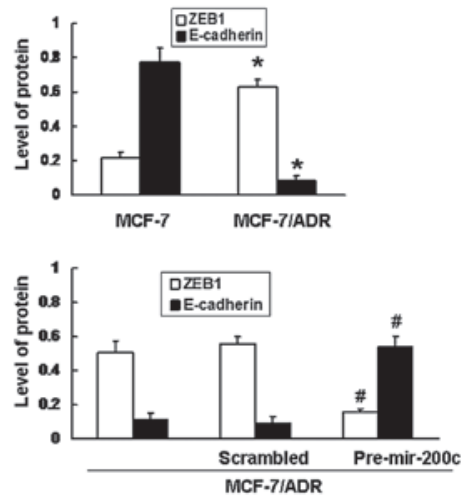

Figure 3. Effect of miRNA-200c on ZEB1 and E-cadherin expression levels in MCF-7/ADR cells. (A) ZEB1 expression is increased in MCF-7/ADR cells, while E-cadherin is decreased. (B) Transfection with pre-mir-200c leads to a decreased level of ZEB1 expression and an increased level of E-cadherin expression in MCF-7/ADR cells. ZEB1 and E-cadherin were measured by western blot analysis and normalized by $\beta$-actin. ${ }^{*} \mathrm{P}<0.01$ compared with MCF-7 cells, ${ }^{\prime} \mathrm{P}<0.01$ compared with scrambled control. ADR, doxorubicin; miRNA-200c, microRNA-200c.

that E-cadherin protein was absent in MCF-7/ADR cells which did not express miRNA-200c but expressed ZEB1 (Fig. 3A). To determine if translational inhibition of ZEB1 restored the expression of E-cadherin, MCF-7/ADR cells were transfected with miRNA-200c precursor. Fig. 3B demonstrates that the ectopic upregulation of miRNA-200c expression levels efficiently reduced the levels of ZEB1 protein in MCF-7/ADR cells. In addition, the downregulation of ZEB1 in miRNA200c-transfected MCF-7/ADR cells was also accompanied by an increase in the level of E-cadherin protein (Fig. 3B). Taken together, our results indicate that the indirect upregulation of E-cadherin may be involved in miRNA-200c-mediated drug sensitivity in MCF-7/ADR cells.

Restoration of miRNA-200c suppressed Akt signaling via E-cadherin-mediated PTEN regulation in MCF-7/ADR cells. PTEN acts downstream of E-cadherin and directly inhibits Akt signaling by inhibiting its phosphorylation. This inhibition is important in tumor cell apoptosis and drug resistance. Thus, we compared the levels of PTEN expression and Akt phosphorylation between the two cell lines. As shown in Fig. 4A, MCF-7/ADR cells with depleted E-cadherin levels exhibited decreased levels of PTEN expression and an enhanced level of phosphorylated Akt. By contrast, forced reintroduction of miRNA-200c precursor upregulated E-cadherin and restored the expression of PTEN, eliminating Akt phosphorylation (Fig. 4B). These results indicate that E-cadherin levels positively correlate with PTEN and inversely correlate with Akt phosphorylation. E-cadherin may additionally inhibit Akt phosphorylation by positively regulating PTEN expression. To further confirm the role of E-cadherin/PTEN/Akt signaling in miRNA-200c-mediated drug resistance, we co-transfected the miRNA-200c precursor and E-cadherin siRNA into MCF-7/ ADR cells. As shown in Fig. 4C, E-cadherin siRNA inhibited the effects of the miRNA-200c precursor on the changes of E-cadherin, PTEN and Akt phosphorylation. Taken together, these data strongly suggest that miRNA-200c increases PTEN expression by indirectly inducing E-cadherin expression, thus
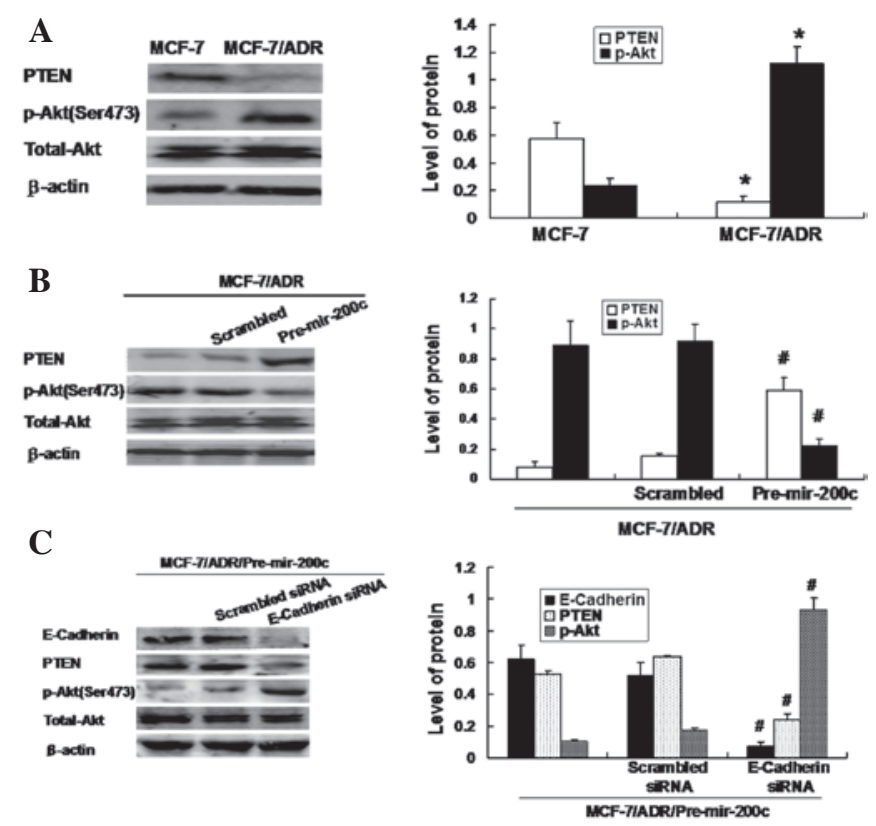

Figure 4. Effect of miRNA-200c on the expression levels of PTEN and Akt phosphorylation (p-Akt) in MCF-7/ADR cells. (A) PTEN expression levels are decreased in MCF-7/ADR cells while p-Akt expression levels are increased. (B) Transfection with pre-mir-200c leads to an increase in the level of PTEN expression and a decrease in p-Akt expression in MCF-7/ADR cells. PTEN, p-Akt, total Akt and $\beta$-actin levels were measured by western blot analysis and normalized by $\beta$-actin and total Akt, respectively. (C) Upregulation of PTEN and inhibition of p-Akt induced by transfection with pre-mir-200c is reversed by E-cadherin siRNA, indicating that E-cadherin is mediated by miRNA-200c-induced PTEN upregulation and p-Akt inhibition. "P<0.01 compared with MCF-7 cells, ${ }^{\text {} P} \mathrm{P}<0.01$ compared with scrambled control. ADR, doxorubicin; miRNA-200c, microRNA-200c.

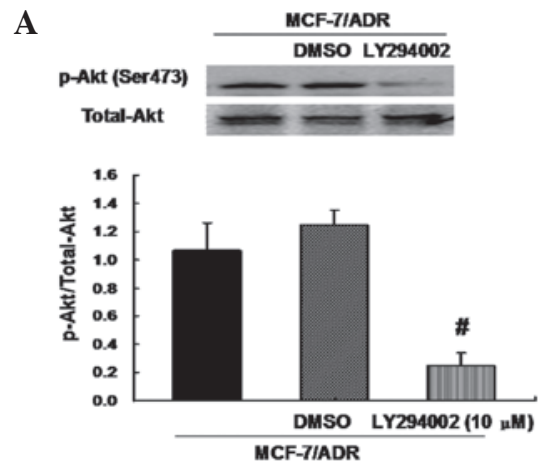

B

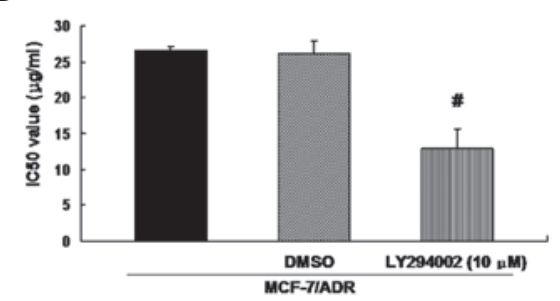

Figure 5. Effect of Akt signaling on ADR sensitivity in MCF-7/ADR cells. (A) LY294002, a specific inhibitor of Akt, eliminated Akt phosphorylation. (B) IC50 values decrease in MCF-7/ADR cells after treatment with LY294002. ${ }^{\#} \mathrm{P}<0.01$ compared with DMSO control. ADR, doxorubicin.

leading to inactivation of Akt signaling by inhibiting its phosphorylation. 
Inhibition of Akt signaling decreased the resistance of MCF-7/ $A D R$ cells to $A D R$. The observation that upregulation of miRNA-200c suppresses Akt phosphorylation and partially restores sensitivity to ADR in MCF-7/ADR cells suggested the possibility that miRNA-200 inhibits drug resistance through inactivation of Akt signaling. To test this hypothesis, we treated MCF-7/ADR cells with fresh medium containing Akt inhibitor LY294002 $(10 \mu \mathrm{M})$ for $24 \mathrm{~h}$, and a control with medium containing $0.1 \%$ dimethyl sulfoxide (DMSO) was used. Treatment with LY294002 eliminated Akt phosphorylation in MCF-7/ADR cells compared with the control (Fig. 5A). LY294002 additionally partially restored sensitivity of MCF-7/ ADR cells to ADR, as shown by the decreased IC50 values (Fig. 5B). These data suggest that miRNA-200c-mediated drug sensitivity of MCF-7/ADR cells is closely correlated with the inactivation of Akt signaling.

\section{Discussion}

ADR exhibits a promising activity against numerous types of cancer, including breast cancer, however, ADR resistance of cancer cells remains a major obstacle for clinical treatment, with its precise mechanism unclear. miRNA-200c has been shown to inhibit tumorigenesis, including EMT, proliferation and metastasis. Emerging evidence suggests that the loss of miRNA-200c expression is a common event in the acquisition of cancer cell resistance to chemotherapeutic drugs, indicating that miRNA-200c is also important in the development of drug resistance of cancer cells. To examine the effects of miRNA-200c on ADR resistance, we tested the expression of miRNA-200c in breast cancer cells with ADR resistance (MCF-7/ADR cells) and observed that miRNA-200c expression was deceased in MCF-7/ADR cells compared with parental MCF-7 cells. However, reintroduction of miRNA200c by transfection with miRNA-200c precursor into MCF-7/ ADR cells markedly enhanced the sensitivity of MCF-7/ADR cells to ADR, suggesting that an inverse correlation between miRNA-200c expression and ADR resistance in breast cancer cells may exist. Similarly, Kovalchuk et al reported that miRNA-200c expression was lower in MCF-7 breast cancer cells which possessed ADR resistance (10) and restoring miRNA-200c levels increased ADR sensitivity through regulation of the p53 apoptotic pathway in breast cancer MDA-MB-231 cells (18). These data demonstrate that the loss of miRNA-200c is a critical determinant for the establishment of an ADR-resistant phenotype in breast cancer.

In breast cancer cells with a mesenchymal phenotype, overexpression of miRNA-200c enhances E-cadherin expression by directly targeting and downregulating ZEB1 expression and indirectly increasing acetylation of histone $\mathrm{H} 3$ at the E-cadherin promoter, resulting in the repression of EMT (18). ZEB1 has been identified as a direct transcriptional repressor of E-cadherin due to its abilitly to directly bind E-box-like sequences in the E-cadherin promoter and thereby repress E-cadherin (17). Furthermore, E-cadherin is one of the key downstream regulators of miRNA-200c contributing to EMT $(16,19)$ and is also important in inhibiting tumor invasion and proliferation, as well as inducing cell apoptosis (20). It has been shown that the expression of E-cadherin is lower in methotrexate-resistant human HT29 colon cancer cells (15), and restoration of E-cadherin expression levels increases the sensitivity of cancer cells to anticancer agents, including etoposide (21), taxol (22) and epidermal growth factor receptor inhibitor $(23,24)$. These findings indicate that E-cadherin expression may be involved in the drug sensitivity of tumor cells. In this study, we have demonstrated that the expression of E-cadherin was decreased in MCF-7/ADR cells compared with MCF-7 cells, while the level of ZEB1 expression was increased. More importantly, upregulation of miRNA-200c expression significantly repressed its direct target ZEB1 and restored E-cadherin levels as well as the sensitivity to ADR. Supporting our data, Park et al reported that miRNA-200c was identified as an epithelial marker in NCI60 human cancer cell lines since it was selectively expressed in only E-cadherinpositive and vimentin-negative cancer cell lines that lack ZEB1 expression, demonstrating a strong correlation between miRNA-200c and ZEB1 and E-cadherin expression levels (13). Our data demonstrate that enforced miRNA-200c expression in MCF-7 breast cancer cells causes the repression of ZEB1, which subsequently increases the expression of E-cadherin, leading to the increased sensitivity of breast cancer cells to ADR.

E-cadherin has been shown to function as an outside-in signaling receptor, transducing signals from the outside to the inside of the cell. Therefore, examining how E-cadherin affects the susceptibility of tumor cells to ADR within cells may provide a better understanding with regard to the development of miRNA-200c-mediated drug resistance in breast cancer cells. PTEN is a dual-specificity phosphatase located in the cytoplasm which is responsible for dephosphorylating lipid and protein substrates (11). Lau et al reported that loss of E-cadherin inhibited ovarian cancer cell growth by repressing Egrl-mediated PTEN transcription (14). Li et al have shown that restoring E-cadherin-mediated cell-cell adhesion improved PTEN protein levels by increasing PTEN protein stability and inhibiting degradation in human breast cancer cells (11). In agreement, we reported in the present study that miRNA-200c-mediated depletion of E-cadherin in MCF-7/ ADR cells was associated with a decrease in PTEN levels as evidence demonstrates that the upregulation of E-cadherin by reintroduction of miRNA-200c precursor restored the expression of PTEN. PTEN may inactivate the Akt signaling pathway by suppressing Akt phosphorylation via dephosphorylating phosphotidylinositol- $(3,4,5)$-triphosphate (the product of PI3K) (25-28). Consistent with this finding, our data showed that there was an increased expression of $\mathrm{p}-\mathrm{Akt}$ level in MCF-7/ADR cells, associated with low levels of PTEN. By contrast, restoring miRNA-200c-mediated PTEN levels in MCF-7/ADR cells by transfection with miRNA-200c precursor reduced p-Akt expression. Specifically, knockdown of miRNA-200c-mediated exogenous expression of E-cadherin reduced the expression levels of E-cadherin and PTEN, and activated Akt phosphorylation again. Therefore, our data suggest that dysregulation of PTEN expression and Akt phosphorylation are crucial in E-cadherin-induced intracellular signaling pathways.

In a study examining ovarian cancer, Lee et al showed that the specific PI3K inhibitor LY294002 sensitized cisplatinresistant cell lines (OVCAR-3/CDDP) to cisplatin-induced apoptosis and decreased cell viability, suggesting that activa- 
tion of the PI3K/Akt signaling may contribute to acquired cisplatin resistance (29). In the present study, we also observed the effects of Akt phosphorylation on ADR resistance and showed that abolishing Akt phosphorylation by LY294002 partially restored the sensitivity of MCF-7/ADR cells to ADR. These data indicate that an inactivated Akt signaling pathway may inhibit the development of drug resistance of breast cancer cells.

In conclusion, we demonstrated that miRNA-200c is important in the development of ADR resistance in breast cancer cells. miRNA-200c increases the drug sensitivity of breast cancer cells to ADR by inactivating the Akt pathway through the E-cadherin-mediated upregulation of PTEN. This study provides novel insights into the mechanisms of drug resistance of tumor cells and highlights miRNA-200c as a new target to improve chemosensitvity.

\section{References}

1. Roberti A, La Sala D and Cinti C: Multiple genetic and epigenetic interacting mechanisms contribute to clonally selection of drugresistant tumors: current views and new therapeutic prospective. J Cell Physiol 207: 571-581, 2006.

2. Glasspool RM, Teodoridis JM and Brown R: Epigenetics as a mechanism driving polygenic clinical drug resistance. Br J Cancer 94: 1087-1092, 2006.

3. Iwasa Y, Nowak MA and Michor F: Evolution of resistance during clonal expansion. Genetics 172: 2557-2566, 2006.

4. Bartel DP: MicroRNAs: genomics, biogenesis, mechanism, and function. Cell 116: 281-297, 2004.

5. Slaby O, Jancovicova J, Lakomy R, et al: Expression of miRNA$106 \mathrm{~b}$ in conventional renal cell carcinoma is a potential marker for prediction of early metastasis after nephrectomy. J Exp Clin Cancer Res 29: 90, 2010.

6. Philippidou D, Schmitt M, Moser D, et al: Signatures of microRNAs and selected microRNA target genes in human melanoma. Cancer Res 70: 4163-4173, 2010.

7. Karakatsanis A, Papaconstantinou I, Gazouli M,Lyberopoulou A Polymeneas $\mathrm{G}$ and Voros D: Expression of microRNAs, miR-21, miR-31, miR-122, miR-145, miR-146a, miR-200c, miR-221, miR-222, and miR-223 in patients with hepatocellular carcinoma or intrahepatic cholangiocarcinoma and its prognostic significance. Mol Carcinog Dec 27, 2011 (Epub ahead of print). doi: $10.1002 / \mathrm{mc} .21864$

8. Cochrane DR, Spoelstra NS, Howe EN, Nordeen SK and Richer JK: MicroRNA-200c mitigates invasiveness and restores sensitivity to microtubule-targeting chemotherapeutic agents Mol Cancer Ther 8: 1055-1066, 2009.

9. Ceppi P, Mudduluru G, Kumarswamy R, et al: Loss of miR-200c expression induces an aggressive, invasive, and chemoresistant phenotype in non-small cell lung cancer. Mol Cancer Res 8: 1207-1216, 2010

10. Kovalchuk O, Filkowski J, Meservy J, et al: Involvement of microRNA-451 in resistance of the MCF-7 breast cancer cells to chemotherapeutic drug doxorubicin. Mol Cancer Ther 7: 2152-2159, 2008.

11. Li Z, Wang L, Zhang W, et al: Restoring E-cadherin-mediated cell-cell adhesion increases PTEN protein level and stability in human breast carcinoma cells. Biochem Biophys Res Commun 363: 165-170, 2007.
12. Kim SM, Kim JS, Kim JH, et al: Acquired resistance to cetuximab is mediated by increased PTEN instability and leads cross-resistance to gefitinib in HCC827 NSCLC cells. Cancer Lett 296: 150-159, 2010.

13. Park SM, Gaur AB, Lengyel E and Peter ME: The miR-200 family determines the epithelial phenotype of cancer cells by targeting the E-cadherin repressors ZEB1 and ZEB2. Genes Dev 22: 894-907, 2008.

14. Lau MT, Klausen C and Leung PC: E-cadherin inhibits tumor cell growth by suppressing PI3K/Akt signaling via $\beta$-cateninEgrl-mediated PTEN expression. Oncogene 30: 2753-2766, 2011.

15. Selga E, Morales C, Noé V, Peinado MA and Ciudad CJ: Role of caveolin 1, E-cadherin, Enolase 2 and PKCalpha on resistance to methotrexate in human HT29 colon cancer cells. BMC Med Genomics 1: 35, 2008.

16. Hurteau GJ,Carlson JA,Spivack SD and Brock GJ: Overexpression of the microRNA hsa-miR-200c leads to reduced expression of transcription factor 8 and increased expression of E-cadherin. Cancer Res 67: 7972-7976, 2007.

17. Eger A, Aigner K, Sonderegger S, et al: DeltaEF1 is a transcriptional repressor of E-cadherin and regulates epithelial plasticity in breast cancer cells. Oncogene 24: 2375-2385, 2005.

18. Tryndyak VP, Beland FA and Pogribny IP: E-cadherin transcriptional down-regulation by epigenetic and microRNA-200 family alterations is related to mesenchymal and drug-resistant phenotypes in human breast cancer cells. Int J Cancer 126: 2575-2583, 2010.

19. Hurteau GJ, Spivack SD and Brock GJ: Potential mRNA degradation targets of hsa-miR-200c, identified using informatics and qRT-PCR. Cell Cycle 5: 1951-1956, 2006.

20. Junxia W, Ping G, Yuan H, et al: Double strand RNA-guided endogeneous E-cadherin up-regulation induces the apoptosis and inhibits proliferation of breast carcinoma cells in vitro and in vivo. Cancer Sci 101: 1790-1796, 2010.

21. Sasaki CY, Lin HC and Passaniti A: Expression of E-cadherin reduces bcl-2 expression and increases sensitivity to etoposideinduced apoptosis. Int J Cancer 86: 660-666, 2000.

22. Ferreira P, Oliveira MJ, Beraldi E, et al: Loss of functional E-cadherin renders cells more resistant to the apoptotic agent taxol in vitro. Exp Cell Res 310: 99-104, 2005.

23. Black PC, Brown GA, Inamoto T, et al: Sensitivity to epidermal growth factor receptor inhibitor requires E-cadherin expression in urothelial carcinoma cells. Clin Cancer Res 14: 1478-1486, 2008.

24. Witta SE, Gemmill RM, Hirsch FR, et al: Restoring E-cadherin expression increases sensitivity to epidermal growth factor receptor inhibitors in lung cancer cell lines. Cancer Res 66: 944-950, 2006.

25. Vivanco I and Sawyers CL: The phosphatidylinositol 3-kinase AKT pathway in human cancer. Nat Rev Cancer 2: 489-501, 2002.

26. Oki E, Baba H, Tokunaga E, et al: Akt phosphorylation associates with LOH of PTEN and leads to chemoresistance for gastric cancer. Int J Cancer 117: 376-380, 2005.

27. Stassi G, Garofalo M, Zerilli M, et al: PED mediates AKT-dependent chemoresistance in human breast cancer cells. Cancer Res 65: 6668-6675, 2005.

28. Myers MP, Pass I, Batty IH, et al: The lipid phosphatase activity of PTEN is critical for its tumor supressor function. Proc Natl Acad Sci USA 95: 13513-13518, 1998.

29. Lee S, Choi EJ, Jin C and Kim DH: Activation of PI3K/Akt pathway by PTEN reduction and PIK3CA mRNA amplification contributes to cisplatin resistance in an ovarian cancer cell line. Gynecol Oncol 97: 26-34, 2005. 